\title{
Sobre o problema da precificação livre de inveja na indústria de entretenimento esportivo
}

\author{
Marcos Salvatierra $^{1}$, Rosiane de Freitas ${ }^{1}$ \\ ${ }^{1}$ Instituto de Computação - Universidade Federal do Amazonas (UFAM) \\ marcosms, rosianeeicomp.ufam.br
}

\begin{abstract}
In this paper, we discuss the feasibility of applying the envy-free pricing problem as strategy in modeling the sale of tickets for sporting events. A mathematical formulation in integer nonlinear programming of the problem is proposed, where assumes that the products have different price ranges, that the customers'valuations of the products are given by reservation prices, that consumers are grouped into segments, that the supply of each product is limited, that consumers can claim several units of the same product and also that there is no price difference for the same product. The goal is to maximize the seller's revenue, but in a way that there is buyers' satisfaction. Preliminary computational experiments demonstrate the viability of the proposed formulation.
\end{abstract}

Resumo. Neste trabalho, se analisa a viabilidade de aplicação do problema da precificação livre de inveja como estratégia na modelagem da venda de ingressos para eventos esportivos. Uma formulação matemática em programação não-linear inteira do problema é proposta, onde se assume que as valorações dos produtos atribuídas pelos consumidores se dão pelos preços de reserva, que a oferta de cada produto é limitada, que os consumidores podem pleitear várias unidades de um mesmo produto e, também, que não há diferença de preços para um mesmo produto. O objetivo é maximizar o lucro do vendedor, mas de maneira que haja satisfação dos compradores. Experimentos computacionais preliminares demonstram a viabilidade da formulação proposta.

\section{1 - Introdução}

As mudanças repentinas que vêm ocorrendo no mercado apresentam desafios às companhias exigindo destas a elaboração de novas ideias inovadoras e produtivas a fim de manter a competitividade e a rentabilidade. Um segmento que encontra-se no bojo das discussões de mercado na atualidade é a indústria de entretenimento esportivo, que movimenta bilhões de dólares anualmente. No processo de obtenção de uma estimativa do volume de movimentação financeira deste segmento, uma série de fatores são levados em conta, como a venda de ingressos, produtos e artigos esportivos, acordos de transmissão, patrocínios, entre outros. Os resultados apresentados neste artigo têm como foco o aspecto da venda de ingressos, que é a mais tradicional fonte de renda na indústria do esporte.

Motivado pela realização do maior evento esportivo deste ano, a Copa do Mundo da Rússia de 2018, este trabalho visa apresentar uma formulação matemática em programação não-linear inteira mista do problema da precificação livre de inveja aplicada à venda de ingressos de jogos de futebol. 
Para se alcançar este objetivo, primeiramente será apresentado o problema da precificação livre de inveja na venda de ingressos esportivos. Em seguida, será feita uma formulação MINLP (Mixed-Integer Nonlinear Programming) seguido de uma proposta de linearização para o caso hipotético da venda de ingressos para a Copa do Mundo da Rússia 2018. Por fim, serão apresentados e discutidos resultados de algumas simulações.

\section{2 - O problema da precificação livre de inveja na venda de ingressos esportivos}

No problema da precificação livre de inveja [Guruswami et al. 2005], considera-se como dados de entrada um conjunto de consumidores, um conjunto de produtos e uma valoração atribuída a cada produto por cada consumidor, produzindo como saídas uma precificação e uma alocação livres de inveja, ou seja, ao final espera-se que cada comprador esteja tão satisfeito com a alocação atribuída a ele quanto qualquer outra, levando em consideração a utilidade de cada produto para cada comprador. De maneira formal, tem-se como entrada um conjunto $I=\{1, \ldots, m\}$ de compradores, um conjunto $J=\{1, \ldots n\}$ de produtos e uma valoração $v \in \mathbb{R}_{+}^{m \times n}$ em que $v_{i j}$ é a valoração atribuída pelo consumidor $i$ ao produto $j$. As saídas são uma precificação $\pi \in \mathbb{R}_{+}^{n}$ em que $\pi_{j}$ é o preço do produto $j$ e uma alocação $X \in\{0,1\}^{m \times n}$ em que $x_{i j}=1$ se o consumidor $i$ compra o produto $j$ e $x_{i j}=0$, caso contrário. Assim, a receita total do vendedor é dada por $\sum_{i=1}^{m} \sum_{j=1}^{n} x_{i j} \pi_{j}$ e o problema busca maximizar esta receita, respeitando a satisfação dos compradores (no sentido da compra livre de inveja), dada pela não negatividade (ou excedente) do comprador $i$ para o produto $j: e_{i j}=v_{i j}-\pi_{j} \geq 0$.

\section{3 - O caso hipotético da venda de ingressos para a Copa do Mundo da Rússia 2018}

\section{1 - Formulação MINLP}

Para fins de motivação e desenvolvimento de um protótipo, suponhamos que a empresa responsável pelo evento quer vender ingressos para uma partida entre duas seleções. Uma proposta de modelagem matemática para este fim pode levar em consideração os seguintes aspectos: faixas de preço (os ingressos são tipificados e valorizados de acordo com a proximidade e visibilidade dos assentos em relação ao campo); determinação de valorações (a empresa recorre a análises de mercado que observam o comportamento de compra de potenciais consumidores, estimando as valorações que os consumidores atribuem aos vários tipos de ingresso como sendo os preços de reserva [Farris et al. 2012]); segmentos de consumidores (consumidores com comportamentos de compra semelhantes são agrupados em segmentos); demanda múltipla (os consumidores estão dispostos a adquirir várias unidades de um mesmo tipo de ingresso); oferta limitada (existe um número máximo de unidades disponíveis para cada tipo de ingresso, de acordo com a lotação máxima do estádio); preço não diferenciado (consumidores que desejam o mesmo tipo de ingresso pagam o mesmo preço por ele); compra livre de inveja (cada consumidor se sente pelo menos tão feliz com a alocação atribuída a ele quanto com qualquer outra, considerando a precificação escolhida).

Feitas estas considerações, um modelo matemático que maximiza a receita da empresa ao mesmo tempo que satisfaz o desejo dos consumidores (no sentido da precificação 
livre de inveja) é:

$$
\begin{aligned}
\max \sum_{i=1}^{m} \sum_{j=1}^{n} N_{i} x_{i j} c_{i j} \pi_{j} & \\
\text { s. a. } \quad \sum_{i=1}^{m} x_{i j} c_{i j} & \leq u_{j}, \forall j \in J \\
\sum_{i=1}^{m} N_{i} x_{i j} c_{i j} & \leq T_{j}, \forall j \in J \\
\left(r_{i j}-\pi_{j}\right) x_{i j} & \geq\left(r_{i k}+\delta_{i}\right) x_{i j}-\pi_{k}, \forall i \in I, \forall j, k \in J, j \neq k \\
\left(r_{i j}-\pi_{j}\right) x_{i j} & \geq 0, \forall i \in I, \forall j \in J \\
\pi_{j} & \geq 0, \forall j \in J \\
x_{i j} & \in\{0,1\}, \forall i \in I, \forall j \in J
\end{aligned}
$$

em que: $I=\{1, \ldots, m\}$, é o conjunto de segmentos de consumidores; $N_{i}$ é o número de consumidores do segmento $i ; J=\{1, \ldots, n\}$ é o conjunto de tipos de ingresso; $u_{j}$ é o número de unidades disponíveis do tipo $j$ para cada consumidor; $T_{j}$ é o total de ingressos do tipo $j$ disponíveis; $r_{i j}$ é a valoração (preço de reserva) que os consumidores do segmento $i$ atribuem ao ingresso do tipo $j ; c_{i j}$ é o número unidades do tipo $j$ que os consumidores do segmento $i$ desejam; $\pi_{j}$ é o preço a ser determinado para o ingresso do tipo $j ; \delta_{i}$ é um parâmetro que determina o desempate nos casos em que consumidores possuem mesma utilidade máxima para produtos diferentes [Shioda et al. 2011]; $x_{i j}$ é a variável de decisão que determinará se os consumidores do segmento $i$ adquirirão o ingresso do tipo $j$.

\section{3 .2 - Proposta de linearização}

A fim de linearizar o modelo acima, será introduzida uma variável auxiliar contínua $p$ tal que $p_{i j}=\pi_{j}$, se $x_{i j}=1$, e $p_{i j}=0$ caso contrário, e será considerado também $\bar{r}_{j}=\max _{i}\left\{r_{i j}\right\}$. Assim, o modelo linear correspondente é:

$$
\begin{aligned}
\max \sum_{i=1}^{m} \sum_{j=1}^{n} N_{i} c_{i j} p_{i j} & \\
\text { s. a. } \quad \sum_{i=1}^{m} x_{i j} c_{i j} & \leq u_{j}, \forall j \in J \\
\sum_{i=1}^{m} N_{i} x_{i j} c_{i j} & \leq T_{j}, \forall j \in J \\
\sum_{j \neq k}\left(r_{i j} x_{i j}-p_{i j}\right) & \geq\left(r_{i k}+\delta_{i}\right)\left(\sum_{j \neq k} x_{i j}\right)-\pi_{k}, \forall i \in I, \forall k \in J \\
r_{i j} x_{i j}-p_{i j} & \geq 0, \forall i \in I, \forall j \in J \\
p_{i j} & \geq \pi_{j}-\bar{r}_{j}\left(1-x_{i j}\right), \forall i \in I, \forall j \in J \\
p_{i j} & \leq \pi_{j}, \forall i \in I, \forall j \in J \\
\pi_{j}, p_{i j} & \geq 0, \forall j \in J \\
x_{i j} & \in\{0,1\}, \forall i \in I, \forall j \in J
\end{aligned}
$$

\section{4 - Resultados e discussão}

Os resultados preliminares obtidos indicam a viabilidade da formulação, suportados por experimentos computacionais usando o solver GLPK v. 4.57. 
Em um cenário hipotético, considerou-se um conjunto de oito segmentos de consumidores e quatro tipos de ingresso, sendo que a disponibilidade de todos os tipos era de duas unidades por consumidor, $\operatorname{com} c_{i j}=1 \forall i \leq 4$ e $c_{i j}=2 \forall i \geq 5, N_{i}=10.000 \forall i$, $\delta_{i}=40 \forall i, T_{j}=10.000 \forall j \leq 3$ e $T_{4}=20.000$. Ao final da simulação, a receita do vendedor foi de $\$ 38.900 .000$, o que verifica a maximização da receita observada a condição livre de inveja para os compradores, como mostram as Figuras 1 e 2.

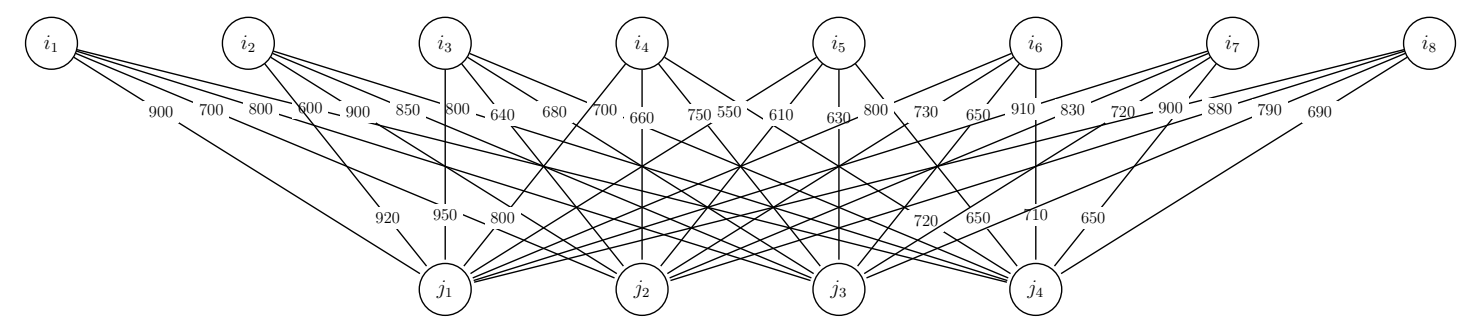

Figura 1. Valorações.

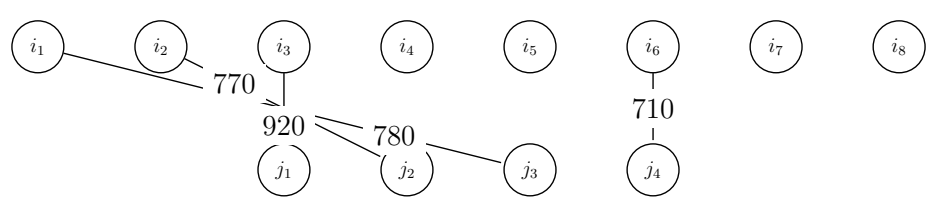

Figura 2. Alocação e precificação.

\section{5 - Conclusões}

Neste trabalho foram apresentados os resultados iniciais da aplicação de um modelo de precificação livre de inveja no âmbito da indústria de entretenimento esportivo. Em andamento, está sendo feita uma análise de sensibilidade dos parâmetros envolvidos e definindo-se instâncias maiores para testes computacionais robustos. Com base nos experimentos iniciais realizados, percebe-se que para um consumidor $i$ que deseja $n$ cópias de um item, sendo que a disponibilidade de unidades do item é menor que $n$, esse consumidor fica sem ser atendido, o que parece ser algo similar ao problema de precificação livre de inveja com objetivo único [Cheung and Swamy 2008]. Dessa forma, pretendemos elaborar formulações em que estes clientes possam ter suas demandas parcialmente aceitas.

\section{Referências}

Cheung, M. and Swamy, C. (2008). Approximation algorithms for single-minded envyfree profit- maximization problems with limited supply. In 49th Annual IEEE Symposium on Foundations of Computer Science, pages 35-44.

Farris, P. W., Bendle, N. T., Pfeifer, P. E., and Reibstein, D. J. (2012). Métricas de marketing - o guia definitivo de avaliação de desempenho de marketing. Bookman.

Guruswami, C., Hartline, J. D., Karlin, A. R., Kempe, D., Kenyon, C., and Mcsherry, F. (2005). On profit-maximizing envy-free pricing. In 18th Annual ACM-SIAM Symposium on Discrete Algorithms, 2005, Vancouver, pages 1164-1173. SIAM.

Shioda, R., Tuncel, L., and Myklebust, T. G. J. (2011). Maximum utility product pricing models and algorithms based on reservation price. Computational Optimization and Applications, 48(2):157-198. 